\title{
The Ising Model and Percolation on Trees and Tree-Like Graphs
}

\author{
Russell Lyons* \\ Department of Mathematics, Stanford University, Stanford, CA 94305-2125, USA
}

\begin{abstract}
We calculate the exact temperature of phase transition for the Ising model on an arbitrary infinite tree with arbitrary interaction strengths and no external field. In the same setting, we calculate the critical temperature for spin percolation. The same problems are solved for the diluted models and for more general random interaction strengths. In the case of no interaction, we generalize to percolation on certain tree-like graphs. This last calculation supports a general conjecture on the coincidence of two critical probabilities in percolation theory.
\end{abstract}

\section{Introduction}

Consider a tree, as in Fig. 1; we use the word tree to mean a countable connected graph which has no loops or cycles and which is locally finite (i.e., each vertex belongs only to a finite number of edges). In the Ising model of ferromagnetism [KS, Pr1, Big], there is a particle at each vertex with spin either up $(+1)$ or down $(-1)$. Each particle interacts with its nearest neighbors in such a way as to favor alignment of the spins; we shall assume that there is no external magnetic field. At temperatures higher than a certain critical temperature, $T_{c}$, there is only one Gibbs state, while at temperatures below $T_{c}$, there are at least two. (In fact, for $T<T_{\text {c }}$, there are an uncountable number of extreme Gibbs states on a tree.) Clearly, adding edges and vertices to a tree can only increase its critical temperature [Lig, Theorem IV.1.21, p. 186]. We shall at first assume that the interaction strength is the same along all edges. Thus, $T_{c}$ is a measure of the number of edges per vertex "on average." Remarkably, after a scale conversion, this notion of average number of edges per vertex coincides with one which has already been studied in connection with percolation, random walks, and Hausdorff dimension [Lyo]. This correspondence between the Ising model and percolation, exact for trees, is only approximate for other graphs [Bis1, Bis3].

\footnotetext{
* Research partially supported by an NSF Mathematical Sciences Postdoctoral Research Fellowship
} 


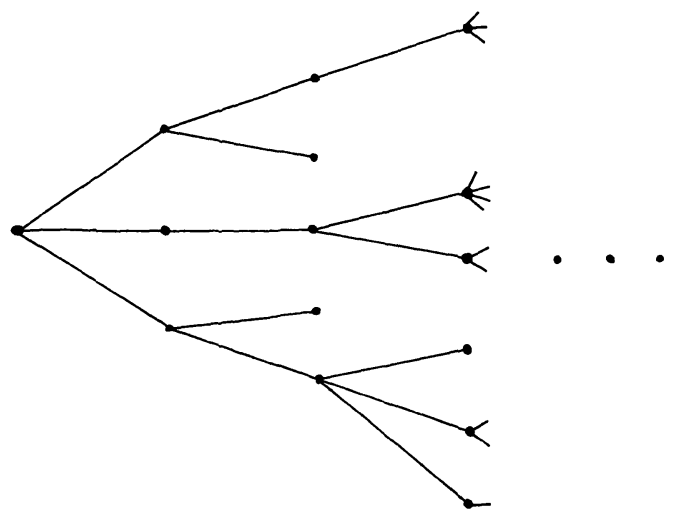

Fig. 1.

There is another critical temperature, $T_{p c}$, above which the probability of finding an infinite (connected) cluster of (aligned) spins is 0 for any Gibbs state, while below $T_{p c}$ this probability is 1 for some Gibbs state. We shall calculate $T_{p c}$ as well and show that it depends only on $T_{c}$.

In the (quenched) diluted Ising model, particles are removed from the tree at random and independently, so that each particle remains with some fixed probability $p \in[0,1]$. In this situation, $T_{c}(p)$ and $T_{p c}(p)$ are random variables, but by Kolmogorov's $0-1$ law, they are constant almost surely. In fact, the value of this constant is easily calculated by combining the results mentioned above with the percolation results in $[\mathbf{L y o}]$.

All of these calculations can be generalized to the case of differing interaction strengths along different edges and even to random interaction strengths. Past results for the Ising model on trees have been exclusively for homogeneous trees (where each vertex has the same number of edges as every other) [Pr1, KS, Spi, MS, KT, Mtb, Mtd, KM, Con].

In order to obtain the percolation results for the Ising model, we first study a general percolation model. This extends a result of [Lyo] and also enables us to study percolation on tree-like graphs, as in Fig. 2. Here, we call a graph tree-like if it is countable, connected, locally finite, and each vertex belongs only to a finite number of cycles (simple closed paths). Under mild assumptions, we show that the critical probability, $p_{c}$, for Bernoulli percolation is equal to another more easily calculated critical value, $p_{\text {cut }}$. This lends further support to the conjecture made in [Lyo] that $p_{c}=p_{\text {cut }}$ for all countable graphs. Previous calculations of $p_{c}$ for tree-like graphs, other than trees, have been for regular structures [FE, Bis2, Con].

We now describe our results more precisely. Given a tree, $\Gamma$, designate one of its vertices as the root, 0 . (None of our results depend on which vertex this is.) If $\sigma$ is a vertex, we write $|\sigma|$ for the number of edges on the shortest path from 0 to $\sigma$. A cutset, $\Pi$, is a finite set of vertices such that every infinite path (i.e., path containing infinitely many distinct vertices) from 0 intersects $\Pi$. The branching 


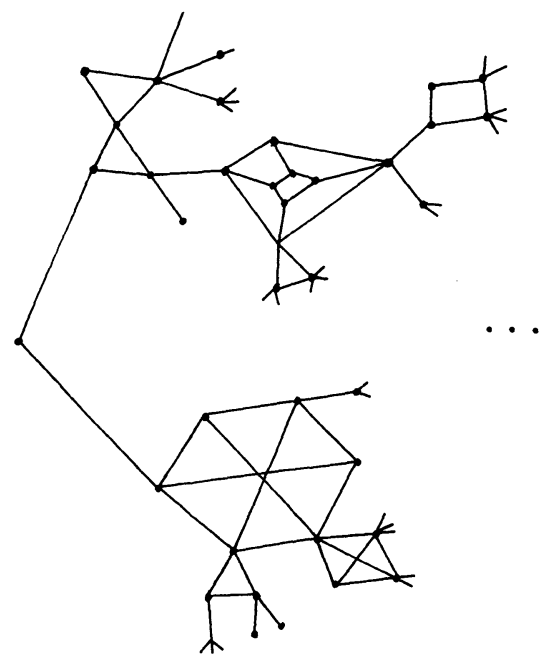

Fig. 2.

number of $\Gamma[$ Lyo $]$, denoted br $\Gamma$, is defined by

$$
\operatorname{br} \Gamma=\inf \left\{\lambda>0: \inf _{\Pi} \sum_{\sigma \in \Pi} \lambda^{-|\sigma|}=0\right\} \text {. }
$$

As explained in [Lyo], this represents an average number of branches per vertex, where the edge leading towards the root does not count as a branch. This is independent of the chosen root, so that we may regard $1+\mathrm{br} \Gamma$ as an average number of edges per vertex, i.e., an average coordination number.

If $J>0$ is the interaction strength along each bond and $k$ is Boltzmann's constant, then phase transition in the Ising model on $\Gamma$ occurs at the temperature

$$
T_{c}=\frac{J}{k \operatorname{coth}^{-1} \text { br } \Gamma},
$$

as we show in Sect. 2. In the diluted Ising model, we first create a random graph by means of a Bernoulli site percolation process on $\Gamma$, then we consider the Ising model on that random graph. If $p$ is the probability of a vertex of $\Gamma$ remaining, then this is of interest only for $p$ larger than the critical probability, $p_{c}$, which equals (br $\Gamma)^{-1}[\mathbf{L y o}]$. In this case, we have

$$
T_{c}(p)=\frac{J}{k \operatorname{coth}^{-1}(p \cdot \mathrm{br} \Gamma)} \text { a.s. }
$$

The following more general result in Sect. 2 allows for arbitrary interaction strengths. For vertices $\sigma$ and $\tau$, we write $\sigma \leqq \tau$ if $\sigma$ is on the shortest path from 0 to $\tau$. If $\sigma \neq 0$, then $\overleftarrow{\sigma}$ denotes the vertex such that $\overleftarrow{\sigma} \leqq \sigma$ and $|\overleftarrow{\sigma}|=|\sigma|-1$. The edge from $\bar{\sigma}$ to $\sigma$ is denoted $e(\sigma)$. If the interaction strength along $e(\sigma)$ is $J_{\sigma}, p$ is 
the survival probability of each vertex ( $p=1$ for the undiluted model), and

$$
\inf _{0 \neq \sigma \in \Gamma} J_{\sigma}>0, \sup _{0 \neq \sigma \in \Gamma} J_{\sigma}<\infty,
$$

then

$$
T_{c}(p)=\inf \left\{T: \inf _{\Pi} \sum_{\sigma \in \Pi} \prod_{0 \neq \tau \leqq \sigma} p \tanh \frac{J_{\tau}}{k T}=0\right\} \text { a.s. }
$$

Still more generally, we may state the following theorem.

Theorem 1.1. Let $\Gamma$ be a tree and let $J_{\sigma}(0 \neq \sigma \in \Gamma)$ be independent random variables, none a.s. zero, satisfying

$$
\inf \left\{J_{\sigma}: J_{\sigma} \neq 0,0 \neq \sigma \in \Gamma\right\}>0 \text { a.s. }
$$

and

$$
\sup _{0 \neq \sigma \in \Gamma} J_{\sigma}<\infty \quad \text { a.s. }
$$

The critical temperature of phase transition for the Ising model on $\Gamma$ with random interaction strengths $J_{\sigma}$ is

$$
T_{c}=\inf \left\{T: \inf \sum_{\sigma \in \Pi} \prod_{0 \neq \tau \leqq \sigma} \mathbf{E}\left[\tanh \frac{J_{\tau}}{k T}\right]=0\right\} \text { a.s. }
$$

In particular, if $J_{\sigma}$ are identically distributed, then $T_{c}$ is a.s. the solution to the equation

$$
\mathbf{E}\left[\tanh \frac{J_{\sigma}}{k T}\right]=(\operatorname{br} \Gamma)^{-1} .
$$

Note that Theorem 1.1 encompasses the aforementioned diluted model: given constants $J_{\sigma}$, we construct independent random variables equal to $J_{\sigma}$ with probability $p$ and to 0 with probability $1-p$. Consideration of the component connected to 0 with nonzero bond strengths shows that the randomness of the bond strengths in Theorem 1.1 is equivalent to the randomness of the sites in the diluted model. (Of course, this is special to trees.)

In Sect. 3, we study a general percolation process on trees, which we term quasi-Bernoulli. It is used in Sect. 4 for spin percolation in the Ising model and in Sect. 5 for Bernoulli percolation on tree-like graphs. Our first result in Sect. 4 is for constant interaction strength $J>0$; here, the critical temperature for spin percolation is

$$
T_{p c}= \begin{cases}\frac{J}{k \operatorname{coth}^{-1}\left(\frac{\mathrm{br} \Gamma}{2-\mathrm{br} \Gamma}\right)} & \text { if } \quad \text { br } \Gamma<2, \\ +\infty & \text { if } \quad \text { br } \Gamma \geqq 2 .\end{cases}
$$


For the diluted model, we have a.s.

$$
T_{p c}(p)= \begin{cases}\frac{J}{k \operatorname{coth}^{-1}\left(\frac{p \cdot \operatorname{br} \Gamma}{2-p \cdot \operatorname{br} \Gamma}\right)} & \text { if } p \cdot \operatorname{br} \Gamma<2, \\ +\infty & \text { if } p \cdot \operatorname{br} \Gamma \geqq 2 .\end{cases}
$$

More generally, with interaction strengths satisfying (1.1), we have

$$
T_{p c}(p)=\inf \left\{T: \inf \sum_{\boldsymbol{\sigma} \in \Pi} \prod_{0 \neq \tau \leqq \sigma} p\left(1+e^{-2 J_{\tau} /(k T)}\right)^{-1}=0\right\} \text { a.s. }
$$

For random interaction strengths, the critical temperature is determined as follows.

Theorem 1.2. Under the same hypotheses as in Theorem 1.1, the critical temperature for percolation in the Ising model on $\Gamma$ is

$$
T_{p c}=\inf \left\{T: \inf _{\Pi} \sum_{0 \neq \tau \leqq \sigma} \mathbf{E}\left[\left(1+e^{-2 J_{\tau} /(k T)}\right)^{-1}\right]=0\right\} \text { a.s. }
$$

Given a countable connected graph $G$ and a point $0 \in G$, a cutset (relative to 0 ) is a finite set of vertices, $\Pi$, such that every infinite path from 0 intersects $\Pi$. Let $G_{0}(\omega)$ be the (random) connected component of 0 for a Bernoulli bond or site percolation process on $G$ with survival parameter $p$. In [Lyo], we defined the critical probability

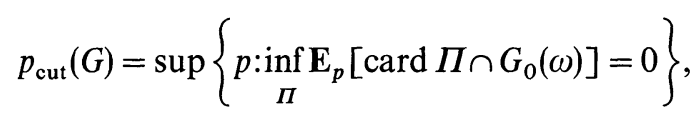

where $\mathbf{E}_{p}$ is expectation with respect to the percolation under consideration and "card" counts the vertices. For comparison, we state the definitions of the usual critical probabilities:

$$
p_{c}(G)=\sup \left\{p: \mathbf{P}_{p}\left[\operatorname{card} G_{0}(\omega)=\infty\right]=0\right\}
$$

and

$$
p_{T}(G)=\sup \left\{p: \mathbf{E}_{p}\left[\operatorname{card} G_{0}(\omega)\right]<\infty\right\},
$$

where $\mathbf{P}_{p}$ denotes probability with respect to the percolation. Clearly

$$
p_{T}(G) \leqq p_{\text {cut }}(G) \leqq p_{c}(G) .
$$

In the case of many regular lattices $G$, all three are equal [AB, MMS]. For arbitrary trees, $\Gamma, p_{\text {cut }}(\Gamma)=p_{c}(\Gamma)$, while $p_{T}(\Gamma)=p_{c}(\Gamma)$ only for sufficiently "regular" $\Gamma$ [Lyo]. We conjectured in [Lyo] that $p_{\text {cut }}(G)=p_{c}(G)$ for all $G$. In Sect. 5, we show that this is the case when $G$ is tree-like and satisfies a mild regularity condition; this condition includes the case when the blocks of $G$ are uniformly bounded in size. Here, a block of $G$ is a maximal connected subgraph having at least one edge and no cutpoints [Har, p. 26], where a cutpoint is a vertex whose removal would disconnect the subgraph. 


\section{The Ising Model}

Given a tree $\Gamma$ and vertices $\sigma, \tau \in \Gamma$, we write $\sigma<\tau$ if $\sigma \leqq \tau$ and $\sigma \neq \tau$. If $\sigma=\bar{\tau}$, we write $\sigma \rightarrow \tau$ and we call $\tau$ a successor of $\sigma$. We shall modify the definition of cutset, $\Pi$, so as to exclude the possibility that $\Pi$ contains two vertices, $\sigma$ and $\tau$, with $\sigma<\tau$. A special cutset is the sphere of radius $n, S_{n}=\{\sigma \in \Gamma:|\sigma|=n\}$. We write $\sigma \leqq \Pi$ if every infinite path from $\sigma$ intersects $\Pi$; the set of such $\sigma$ is denoted $\leqq \Pi$. We also write $\sigma<\Pi$ if $\sigma \leqq \Pi$ and $\sigma \notin \Pi$. We say that a sequence of cutsets, $\left\{\Pi_{n}\right\}$, tends to $\infty$ if $\lim _{n \rightarrow \infty} \min \left\{|\sigma|: \sigma \in \Pi_{n}\right\}=\infty$. We denote the subtree $\{\tau \in \Gamma: \sigma \leqq \tau\}$ by $\Gamma^{\sigma}$.

Let $\Pi$ be a cutset. Let $u_{\sigma} \in\{+1,-1\}$ be random variables denoting the spins for $\sigma \leqq \Pi$. For "plus" boundary conditions, these are determined by the Hamiltonian

$$
\mathscr{H}_{\Pi}(u)=-\frac{J}{k T} \sum_{0<\sigma \leqq \Pi} u_{\sigma} u_{\bar{\sigma}}
$$

in accordance with Boltzmann's equation

$$
\mathbf{P}_{\Pi}[u \in A]=\sum_{u \in A} e^{-\mathscr{H}_{\Pi}(u)} / Z^{\Pi} \quad(A \subseteq \mathscr{U}),
$$

where

$$
Z^{\Pi}=\sum_{u \in \mathscr{U}} e^{-\mathscr{H}_{\Pi}^{(u)}}
$$

is the partition function and $\mathscr{U}=\left\{u \in\{ \pm 1\}^{\leq}:\left.u\right|_{\Pi}=+1\right\}$ is the space of all configurations. We are particularly interested in $\mathbf{P}_{\Pi}\left[u_{0}=+1\right]$. For $a= \pm 1$, let

$$
Z_{a}^{\Pi}(0)=\sum_{\substack{u \in \mathscr{C}_{a} \\ u_{0}=a}} e^{-\mathscr{H}_{\Pi}(u)}
$$

More generally, for $\sigma<\Pi$, consider $\sigma$ as the root of $\Gamma^{\sigma}$ and $\Pi \cap \Gamma^{\sigma}$ as a cutset of $\Gamma^{\sigma}$, and define $Z_{a}^{\Pi}(\sigma)$ analogously to $Z_{a}^{\Pi}(0)$ above. Finally, for $\sigma \in \Pi$, let

$$
Z_{a}^{\Pi}(\sigma)=\delta_{a,+1}
$$

Thus,

$$
\begin{aligned}
Z_{a}^{\Pi}(0) & =\sum_{\substack{u \in U_{a} \\
u_{0}=a}} \prod_{\sigma \in S_{1}}\left[e^{a u_{\sigma} J(k T)} \exp \left\{\frac{J}{k T} \sum_{\sigma<\tau \leqq \Pi} u_{\tau} u_{\bar{\tau}}\right\}\right] \\
& =\sum_{v \in\{ \pm 1\}_{1}^{\mathbf{s}_{1}}} \prod_{\sigma \in S_{1}} e^{a v_{\sigma} J /(k T)} Z_{v_{\sigma}}^{\Pi}(\sigma) \\
& =\prod_{\sigma \in S_{1}}\left[e^{a J /(k T)} Z_{+1}^{\Pi}(\sigma)+e^{-a J /(k T)} Z_{-1}^{\Pi}(\sigma)\right],
\end{aligned}
$$

whence

$$
\begin{aligned}
\frac{\mathbf{P}_{\Pi}\left[u_{0}=+1\right]}{\mathbf{P}_{\Pi}\left[u_{0}=-1\right]} & =\frac{Z_{+1}^{\Pi}(0) / Z^{\Pi}}{Z_{-1}^{\Pi}(0) / Z^{\Pi}}=\prod_{\sigma \in S_{1}} \frac{e^{J /(k T)} Z_{+1}^{\Pi}(\sigma)+e^{-J /(k T)} Z_{-1}^{\Pi}(\sigma)}{e^{-J /(k T)} Z_{+1}^{\Pi}(\sigma)+e^{J /(k T)} Z_{-1}^{\Pi}(\sigma)} \\
& =\prod_{\sigma \in S_{1}} g_{\alpha}\left(Z_{+1}^{\Pi}(\sigma) / Z_{-1}^{\Pi}(\sigma)\right),
\end{aligned}
$$


where $\alpha=e^{2 J /(k T)}$ and

$$
g_{\alpha}(x)=\frac{\alpha x+1}{x+\alpha}
$$

For $\sigma \leqq \Pi$, set

$$
f_{\Pi}(\sigma)=Z_{+1}^{\Pi}(\sigma) / Z_{-1}^{\Pi}(\sigma),
$$

which we interpret as $+\infty$ for $\sigma \in \Pi$. We have thus arrived at a recursion formula for $f_{\Pi}$ :

$$
f_{\Pi}(\sigma)=\left\{\begin{array}{lll}
\prod_{\sigma \rightarrow \tau} g_{\alpha}\left(f_{\Pi}(\tau)\right) & \text { if } & \sigma<\Pi, \\
+\infty & \text { if } & \sigma \in \Pi
\end{array}\right.
$$

The empty product is defined to be 1 , so that it follows from (2.1) that when $\Pi \cap \Gamma^{\sigma}=\varnothing, f_{\Pi}(\sigma)=1$, as it should.

We now pass to the thermodynamic limit. Choose a sequence of cutsets $\left\{\Pi_{n}\right\}$ tending to infinity. From considerations of monotonicity, it is well known that

$$
f(\sigma)=\lim _{\substack{n \rightarrow \infty \\ \sigma \leqq \Pi_{n}}} f_{\Pi_{n}}(\sigma)
$$

exists and is independent of the choice of $\left\{\Pi_{n}\right\}$; furthermore,

$$
f(0)=\frac{\mathbf{P}\left[u_{0}=+1\right]}{\mathbf{P}\left[u_{0}=-1\right]}
$$

the probabilities being taken with respect to the limiting Gibbs state. For $T>T_{c}$, $f(0)=1$, while for $T<T_{c}, f(0)>1$ [Pr1, Proposition 8.9, p. 67].

Theorem 2.1. Let $\Gamma$ be a tree. Phase transition in the Ising model on $\Gamma$ with constant interaction strength $J>0$ occurs at

$$
T_{c}=\frac{J}{k \operatorname{coth}^{-1} \mathrm{br} \Gamma}
$$

If $\beta=(\alpha-1) /(\alpha+1)$, then (2.2) is equivalent to

$$
\beta_{c} \cdot \operatorname{br} \Gamma=1 \text {. }
$$

The key to estimating $f$ lies in replacing $g_{\alpha}$ by an exponential function, as the latter behaves much more simply under composition and multiplication. For this purpose, we shall use the following inequalities.

Lemma 2.2. For $x \geqq 1$,

$$
x^{(\alpha-x) /(\alpha+x)} \leqq g_{\alpha}(x) \leqq x^{\beta} .
$$

Proof. First, by the weighted form of the arithmetic-geometric mean inequality, we have

$$
g_{\alpha}(x)=\frac{\alpha}{\alpha+x} x+\frac{x}{\alpha+x} x^{-1} \geqq x^{\alpha /(\alpha+x)} x^{-x /(\alpha+x)}=x^{(\alpha-x) /(\alpha+x)} .
$$


Second, we have

$$
\begin{aligned}
\log g_{\alpha}(x) & =\int_{1}^{x} \frac{d}{d t} \log g_{\alpha}(t) d t=\int_{1}^{x} \frac{\alpha^{2}-1}{(1+\alpha t)(t+\alpha)} d t \\
& =\int_{1}^{x} \frac{\alpha^{2}-1}{\alpha(t-1)^{2}+(\alpha+1)^{2} t} d t \leqq \int_{1}^{x} \frac{\alpha^{2}-1}{(\alpha+1)^{2} t} d t=\log x^{\beta} .
\end{aligned}
$$

Proof of Theorem 2.1. We shall show that if $\beta \cdot \operatorname{br} \Gamma<1$, then $f(0)=1$, while if $f(0)=1$, then $\beta \cdot$ br $\Gamma \leqq 1$. Given a cutset $\Pi$, define $h$ on $\Pi$ recursively by

$$
h(\sigma)=\left\{\begin{array}{lll}
\alpha^{\beta^{-1}} & \text { if } & \sigma \in \Pi, \\
\prod_{\sigma \rightarrow \tau} h(\tau)^{\beta} & \text { if } & \sigma<\Pi .
\end{array}\right.
$$

We claim that for $n$ large enough that $\Pi \subseteq \leqq \Pi_{n}$, we have $f_{\Pi_{n}}(\sigma) \leqq h(\sigma)$ for $\sigma<\Pi$. This is a consequence of the following facts when combined with (2.1): $g_{\alpha}\left(f_{\Pi_{n}}(\sigma)\right) \leqq \alpha=h(\sigma)^{\beta}$ for $\sigma \in \Pi ; g_{\alpha}\left(f_{\Pi_{n}}(\sigma)\right) \leqq f_{\Pi_{n}}(\sigma)^{\beta}$ for $\sigma<\Pi$. In particular, $f(0) \leqq h(0)$. Set $\theta(\sigma)=\beta^{|\sigma|} \log h(\sigma)$. Then $\theta(\sigma)=\sum_{\sigma \rightarrow \tau} \theta(\tau)$ for $\sigma<\Pi$, whence $\theta(0)=\sum_{\sigma \in \Pi} \theta(\sigma)=\sum_{\sigma \in \Pi} \beta^{|\sigma|-1} \log \alpha$. This yields the bound

$$
f(0) \leqq h(0)=e^{\theta(0)}=\alpha^{\beta^{-1} \Sigma_{\sigma \in \Pi^{\beta}}} .
$$

By definition of br $\Gamma$, it follows that if $\beta \cdot$ br $\Gamma<1$, then $f(0)=1$.

Conversely, suppose that $f(0)=1$. Then $f(\sigma)=1$ for all $\sigma \in \Gamma$ by (2.1). Choose $\left.\beta_{0} \in\right] 0, \beta\left[\right.$ and set $\alpha_{0}=\left(1+\beta_{0}\right) /\left(1-\beta_{0}\right)$. Given $n$ so large that $f_{\Pi_{n}}(\sigma) \leqq \alpha / \alpha_{0}$ for $\sigma \in S_{1}$, there is a cutset $\Pi \subseteq \leqq \Pi_{n}$ such that for all $\sigma \leqq \Pi$,

$$
f_{\Pi_{n}}(\sigma) \leqq \alpha / \alpha_{0}
$$

while for all $\sigma \in \Pi$, there is a successor $\tau_{0}$ of $\sigma$ such that $f_{\Pi_{n}}\left(\tau_{0}\right)>\alpha / \alpha_{0}$. Put $a=g_{\alpha}\left(\alpha / \alpha_{0}\right)$. Since $f_{\Pi_{n}} \geqq 1$, we have for $\sigma \in \Pi$,

$$
f_{\Pi_{n}}(\sigma)=\prod_{\sigma \rightarrow \tau} g_{\alpha}\left(f_{\Pi_{n}}(\tau)\right) \geqq g_{\alpha}\left(f_{\Pi_{n}}\left(\tau_{0}\right)\right)>a .
$$

Furthermore, for $x \leqq \alpha / \alpha_{0}$, we have $(\alpha-x) /(\alpha+x) \geqq \beta_{0}$, whence by (2.4) and Lemma 2.2, we obtain

$$
g_{\alpha}\left(f_{\Pi_{n}}(\sigma)\right) \geqq f_{\Pi_{n}}(\sigma)^{\beta_{0}} \quad(\sigma \leqq \Pi) .
$$

If we define $h$ on $\leqq$ recursively by

$$
h(\sigma)=\left\{\begin{array}{lll}
a & \text { if } & \sigma \in \Pi, \\
\prod_{\sigma \rightarrow \tau} h(\tau)^{\beta_{0}} & \text { if } & \sigma<\Pi,
\end{array}\right.
$$

then it follows that $f_{\Pi_{n}}(\sigma) \geqq h(\sigma)$ for $\sigma \leqq \Pi$. In particular,

$$
f_{\Pi_{n}}(0) \geqq h(0)=a^{\Sigma_{\sigma \in \Pi^{\beta}} \beta_{0}^{|\sigma|}} \text {. }
$$

Since $a>1$ and $f_{\Pi_{n}}(0) \rightarrow 1$, it follows that $\inf _{\Pi} \sum_{\sigma \in \Pi} \beta_{0}^{|\sigma|}=0$-in other words, $\beta_{0} \cdot$ br $\Gamma \leqq 1$. Since this is true for all $\beta_{0}<\beta$, we arrive at our desired conclusion: $\beta \cdot$ br $\Gamma \leqq 1$. 
In combination with Corollary 6.3 of [Lyo], this theorem has the following immediate consequence for the diluted Ising model.

Corollary 2.3. Let $\Gamma$ be a tree, $(\operatorname{br} \Gamma)^{-1}<p \leqq 1$, and $J>0$. For the diluted Ising model on $\Gamma$ with survival probability $p$ and constant interaction strength $J$, we have

$$
T_{c}(p)=\frac{J}{k \operatorname{coth}^{-1}(p \cdot \operatorname{br} \Gamma)} \text { a.s. }
$$

We shall now allow the interaction strengths to vary according to the bond. If $J_{\sigma}$ is the strength along $e(\sigma)$, the Hamiltonians take the form

$$
\mathscr{H}_{\Pi}(u)=-\frac{1}{k T_{0}} \sum_{\sigma \leqq \Pi} J_{\sigma} u_{\sigma} u_{\bar{\sigma}} .
$$

Correspondingly, we write $\alpha_{\sigma}=e^{2 J_{\sigma} /(k T)}$ and $\beta_{\sigma}=\left(\alpha_{\sigma}-1\right) /\left(\alpha_{\sigma}+1\right)=\tanh J_{\sigma} /$ $(k T)$. The same reasoning as above leads to the equation

$$
f_{\Pi}(\sigma)=\left\{\begin{array}{lll}
\prod_{\sigma \rightarrow \tau} g_{\alpha_{\tau}}\left(f_{\Pi}(\tau)\right) & \text { if } & \sigma<\Pi, \\
+\infty & \text { if } & \sigma \in \Pi
\end{array}\right.
$$

Theorem 2.4. The critical temperature of phase transition for the Ising model on a tree $\Gamma$ with interaction strengths $J_{\sigma}>0$ satisfies $T_{*} \leqq T_{c} \leqq T^{*}$, where

$$
T_{*}=\inf \left\{T: \inf _{\Pi \sigma \in \Pi} \sum_{\sigma}\left(\tanh \frac{J_{\sigma}}{k T}\right)_{0<\tau \leqq \sigma} \tanh \frac{J_{\tau}}{k T}=0\right\}
$$

and

$$
T^{*}=\inf \left\{T: \underset{\Pi}{\inf } \sum_{\sigma \in \Pi} J_{\sigma} \prod_{0<\tau<\sigma} \tanh \frac{J_{\tau}}{k T}=0\right\} .
$$

Of course, in most cases, $T_{*}=T^{*}$. Certainly this happens if inf $J_{\sigma}>0$.

Proof. Suppose that $\inf _{\Pi} \sum_{\sigma \in \Pi} J_{\sigma} \prod_{0<\tau<\sigma} \beta_{\tau}=0$. Given a cutset $\Pi$, define $h$ on $\leqq \Pi$ by

$$
h(\sigma)=\left\{\begin{array}{lll}
\alpha_{\sigma}^{\beta_{\sigma}^{-1}} & \text { if } & \sigma \in \Pi, \\
\prod_{\sigma \rightarrow \tau} h(\tau)^{\beta_{\tau}} & \text { if } & \sigma<\Pi .
\end{array}\right.
$$

Then as in the proof of Theorem 2.1, $f_{\Pi_{n}}(\sigma) \leqq h(\sigma)$ for $\sigma<\Pi$ and $\Pi \subseteq \leqq \Pi_{n}$. Therefore

$$
f(0) \leqq h(0)=\exp \sum_{\sigma \in \Pi} \frac{2 J_{\sigma}}{k T} \prod_{0<\tau<\sigma} \beta_{\tau},
$$

which entails, by our supposition, that $f(0)=1$. Thus $T_{c} \leqq T^{*}$.

Conversely, suppose that $f(0)=1$. Choose $T^{\prime}>T$ and define $\alpha_{\sigma}^{\prime}, \beta_{\sigma}^{\prime}$ accordingly. Given $n$ sufficiently large, there is a cutset $\Pi \subseteq \leqq \Pi_{n}$ such that

$$
f_{\Pi_{n}}(\sigma) \leqq \alpha_{\sigma} \frac{1-\beta_{\sigma}^{\prime}}{1+\beta_{\sigma}^{\prime}}=\frac{\alpha_{\sigma}}{\alpha_{\sigma}^{\prime}} \quad(\sigma \leqq \Pi)
$$


while each $\sigma \in \Pi$ has a successor $\tau_{0}$ such that $f_{\Pi_{n}}\left(\tau_{0}\right)>\alpha_{\sigma} / \alpha_{\sigma}^{\prime}$. Put $a_{\sigma}=g_{\alpha_{\sigma}}\left(\alpha_{\sigma} / \alpha_{\sigma}^{\prime}\right)$ and note that $a_{\sigma} \geqq\left(\alpha_{\sigma} / \alpha_{\sigma}^{\prime}\right)^{\beta_{\sigma}^{\prime}}$ by Lemma 2.2. Define $h$ on $\leqq \Pi$ by

$$
h(\sigma)=\left\{\begin{array}{lll}
a_{\sigma} & \text { if } & \sigma \in \Pi, \\
\prod_{\sigma \rightarrow \tau} h(\tau)^{\beta_{\tau}^{\prime}} & \text { if } & \sigma<\Pi .
\end{array}\right.
$$

Then $f_{I_{n}}(\sigma) \geqq h(\sigma)$ for $\sigma \leqq \Pi$, so that

$$
\begin{aligned}
f_{\Pi_{n}}(0) & \geqq h(0)=\exp \sum_{\sigma \in \Pi}\left(\log a_{\sigma}\right) \prod_{0<\tau \leqq \sigma} \beta_{\tau}^{\prime} \\
& \geqq \exp \sum_{\sigma \in \Pi} \beta_{\sigma}^{\prime}\left(\log \frac{\alpha_{\sigma}}{\alpha_{\sigma}^{\prime}}\right)_{0<\tau \leqq \sigma} \beta_{\tau}^{\prime} \\
& =\exp \sum_{\sigma \in \Pi} \beta_{\sigma}^{\prime} \frac{2 J_{\sigma}}{k}\left(\frac{1}{T}-\frac{1}{T^{\prime}}\right) \prod_{0<\tau \leqq \sigma} \beta_{\tau}^{\prime} .
\end{aligned}
$$

Our supposition entails, therefore, that $\inf _{\Pi} \sum_{\sigma \in \Pi} \beta_{\sigma}^{\prime} J_{\sigma} \prod_{\tau \leqq \sigma} \beta_{\tau}^{\prime}=0$. Since $T^{\prime}$ is arbitrary, the conclusion $T_{c} \geqq T_{*}$ ensues.

Corollary 2.5. The critical temperature of phase transition for the diluted Ising model on a tree $\Gamma$ with interaction strengths $J_{\sigma}$ satisfying (1.1) and survival probability $p$ is

$$
T_{c}(p)=\inf \left\{T: \inf _{\Pi \sigma \in \Pi 0<\tau \leqq \sigma} p \tanh \frac{J_{\tau}}{k T}=0\right\} \text { a.s. }
$$

Proof. Let $\Gamma_{0}(\omega)$ be the connected component of 0 arising from the Bernoulli percolation on $\Gamma$, let $\Pi(\omega)=\Pi \cap \Gamma_{0}(\omega)$, and define $f_{\Pi(\omega)}(\sigma)$ for $\sigma \in \Gamma_{0}(\omega)$ as before for the tree $\Gamma_{0}(\omega)$. Let $f_{\omega}(0)=\lim _{\Pi \rightarrow \infty} f_{\Pi(\omega)}(0)$. Now $T_{c}(p)$ is a constant (a.s.) and for $T>T_{c}(p), f_{\omega}(0)=1$ a.s., while for $T<T_{c}(p), f_{\omega}(0)>1$ with positive probability.

Suppose that $\left\{\Pi_{n}\right\}$ is a sequence of cutsets tending to infinity such that $\lim _{n \rightarrow \infty} \sum_{\sigma \in \Pi_{n}} \prod_{0<\tau \leqq \sigma} p \beta_{\tau}=0$. Then

$$
\begin{aligned}
\mathbf{E}_{p}\left[\sum_{\sigma \in \Pi_{n}(\omega)} \prod_{0<\tau \leqq \sigma} \beta_{\tau}\right] & =\sum_{\sigma \in \Pi_{n}} \mathbf{P}_{p}\left[\sigma \in \Gamma_{0}(\omega)\right] \prod_{0<\tau \leqq \sigma} \beta_{\tau} \\
& =\sum_{\sigma \in \Pi_{n}} \prod_{0<\tau \leqq \sigma} p \beta_{\tau} \rightarrow 0,
\end{aligned}
$$

whence, by Fatou's lemma,

$$
\varliminf_{n \rightarrow \infty} \sum_{\sigma \in \Pi_{n}(\omega)} \prod_{0<\tau \leqq \sigma} \beta_{\tau}=0 \text { a.s. }
$$

It follows from (the proof of) Theorem 2.4 that $f_{\omega}(0)=1$ a.s., whence $T_{c}(p) \leqq T$ a.s.

On the other hand, suppose that inf $\sum_{\sigma \in \Pi} \prod_{0<\tau \leqq \sigma} p \beta_{\tau}>0$ and choose $T^{\prime}<T$. Define $\beta_{\sigma}^{\prime}$ accordingly; we claim that inf $\sum_{\sigma \in \Pi}\left(T^{\prime} / T\right)^{|\sigma|} \prod_{0<\tau \leqq \sigma} p \beta_{\tau}^{\prime}>0$. For let $F(t)=\log \tanh t$. 
Then if $t>0$,

whence

$$
F^{\prime}(t)=\frac{\operatorname{sech}^{2} t}{\tanh t}=\frac{2}{\sinh 2 t}<\frac{1}{t}
$$

$$
\log \left(\beta_{\tau} / \beta_{\tau}^{\prime}\right)=\int_{J /\left(k T^{\prime}\right)}^{J /(k T)} F^{\prime}(t) d t<\log \left(T^{\prime} / T\right)
$$

Our claim follows.

We next claim that inf $\sum_{\sigma \in \Pi(\omega)} \prod_{0<\tau \leqq \sigma} \beta_{\tau}^{\prime}>0$ with positive probability. Given this, we may conclude from Theorem 2.4 that $T_{c}(p) \geqq T^{\prime}$ with positive probability, hence a.s. Because of our choice of $T^{\prime}$, this entails the relation $T_{c}(p) \geqq T$ a.s., which completes the proof.

The proof of our claim is exactly parallel to that of the second half of Theorem 6.6 of [Lyo]; we merely sketch it. We call a function, $\theta$, on $\Gamma$ a unit flow if $\theta \geqq 0$, $\theta(0)=1$, and $\forall \sigma \in \Gamma \theta(\sigma)=\sum_{\sigma \rightarrow \tau} \theta(\tau)$. Write $\sigma \wedge \tau$ for the vertex farthest from 0 which is $\leqq$ both $\sigma$ and $\tau$. Put $p_{\sigma}=\prod_{0<\tau \leqq \sigma} p \beta_{\tau}^{\prime}$. Using what was established above, we may find, as in the proof of Corollary 4.2 of [Lyo], a unit flow, $\theta$, such that

$$
\sum_{0 \neq \sigma \in \Gamma} \theta(\sigma)^{2} p_{\sigma}^{-1}<\infty
$$

Define

$$
k(\sigma)=\theta(\sigma) p^{-|\sigma|} \mathbf{1}_{\Gamma_{0}(\omega)}(\sigma), \quad \xi_{n}=\sum_{\sigma \in S_{n}} k(\sigma) .
$$

Then $\left\{\xi_{n}\right\}$ is a nonnegative martingale satisfying

$$
\mathbf{E}_{p}\left[\xi_{n}^{2}\right] \leqq \sum_{\sigma, \tau \in S_{n}} \theta(\sigma) \theta(\tau) p^{-|\sigma \wedge \tau|} \leqq \sum_{|\sigma| \leqq n} \theta(\sigma)^{2} p^{-|\sigma|}
$$

This is uniformly bounded in $n$ by virtue of our choice of $\theta$ (and the fact that $\left.p_{\sigma}<p^{|\sigma|}\right)$. Hence $\left\{\xi_{n}\right\}$ has a nonzero limit with positive probability. Set

$$
g(\sigma)=\prod_{0<\tau \leqq \sigma} \beta_{\tau}^{\prime}
$$

and

$$
Y_{n}=\sum_{\sigma, \tau \in S_{n} \cap \Gamma_{0}(\omega)} g(\sigma \wedge \tau)^{-1} k(\sigma) k(\tau) .
$$

Then $\mathbf{E}_{p}\left[Y_{n}\right] \leqq \sum_{|\sigma| \leqq n} \theta(\sigma)^{2} p_{\sigma}^{-1}$; as this is bounded, we obtain ${\underset{n \rightarrow \infty}{\lim }}_{n \rightarrow \infty}<\infty$ a.s. Therefore $\lim _{n \rightarrow \infty} Y_{n} \xi_{n}^{-2}<\infty$ with positive probability, whereupon our claim follows from Lemma 3.2 of [Lyo].

The proof of Theorem 1.1 is exactly parallel; the only significant change that 
needs to be made is to define $k(\sigma)$ as

$$
\theta(\sigma) \prod_{0<\tau \leqq \sigma} \tanh \frac{J_{\tau}}{k T} / \mathbf{E}\left[\tanh \frac{J_{\tau}}{k T}\right] .
$$

\section{Quasi-Bernoulli Percolation}

Given a tree $\Gamma$, suppose that $(\Omega, \mathbf{P})$ is a probability space associated with random subgraphs $\Gamma(\omega)$ of $\Gamma(\omega \in \Omega)$. We assume the measurability of the indicator functions corresponding to the presence or absence in $\Gamma(\omega)$ of any vertex or edge of $\Gamma$. Let $\Gamma_{0}(\omega)$ be the connected component of 0 in $\Gamma(\omega)$ and $K(\sigma)=\left\{\omega: \sigma \in \Gamma_{0}(\omega)\right\}$. We say that $(\Gamma, \Omega, \mathbf{P})$ is a quasi-Bernoulli percolation process if there is a constant $M<\infty$ such that for all $\sigma, \tau \in \Gamma$,

$$
\mathbf{P}[K(\sigma) \cap K(\tau)] \cdot \mathbf{P}[K(\sigma \wedge \tau)] \leqq M \mathbf{P}[K(\sigma)] \mathbf{P}[K(\tau)] .
$$

(The motivation for the name "quasi-Bernoulli" is more apparent when the above inequality is expressed in terms of probabilities conditioned on $K(\sigma \wedge \tau)$.)

Theorem 3.1. Let $(\Gamma, \Omega, \mathbf{P})$ be a quasi-Bernoulli percolation process. If

$$
\inf _{\Pi} \sum_{\sigma \in \Pi} \mathbf{P}[K(\sigma)]=0
$$

then $\mathbf{P}\left[\operatorname{card} \Gamma_{0}(\omega)=\infty\right]=0$. If there are positive numbers $w_{n}$ such that $\sum_{n \geqq 1} w_{n}<\infty$ and

$$
\inf _{\Pi} \sum_{\sigma \in \Pi} w_{|\sigma|} \mathbf{P}[K(\sigma)]>0,
$$

then $\mathbf{P}\left[\operatorname{card} \Gamma_{0}(\omega)=\infty\right]>0$.

Proof. If (3.1) holds, then there is a sequence of cutsets $\left\{\Pi_{n}\right\}$ such that

$$
\mathbf{E}\left[\operatorname{card}\left(\Gamma_{0}(\omega) \cap \Pi_{n}\right)\right]=\sum_{\sigma \in \Pi_{n}} \mathbf{P}[K(\sigma)] \rightarrow 0 .
$$

By virtue of Fatou's lemma,

$$
\varliminf_{n \rightarrow \infty} \operatorname{card}\left(\Gamma_{0}(\omega) \cap \Pi_{n}\right)=0 \quad \text { a.s., }
$$

which is the same as card $\Gamma_{0}(\omega)<\infty$ a.s.

On the other hand, if (3.2) holds, then the max-flow min-cut theorem provides, as in the proof of Corollary 4.2 of [Lyo], a unit flow $\theta$ on $\Gamma$ such that

$$
\sum_{\sigma \in \Gamma} \theta(\sigma)^{2} \mathbf{P}[K(\sigma)]^{-1}<\infty
$$

Define

$$
X_{n}=\sum_{\sigma \in S_{n}} \mathbf{1}_{K(\sigma)} \theta(\sigma) \mathbf{P}[K(\sigma)]^{-1}
$$


Suppose, for a contradiction, that $\Gamma_{0}(\omega)$ is finite a.s. Then $X_{n}$ is eventually 0 a.s. Now

$$
\begin{aligned}
\mathbf{E}\left[X_{n}^{2}\right] & =\sum_{\sigma, \tau \in S_{n}} \theta(\sigma) \theta(\tau) \mathbf{P}[K(\sigma)]^{-1} \mathbf{P}[K(\tau)]^{-1} \mathbf{P}[K(\sigma) \cap K(\tau)] \\
& \leqq M \sum_{\sigma, \tau \in S_{n}} \theta(\sigma) \theta(\tau) \mathbf{P}[K(\sigma \wedge \tau)]^{-1} \\
& =M \sum_{|\psi| \leqq n} \mathbf{P}[K(\psi)]^{-1} \sum_{\substack{\sigma \wedge \tau=\psi \\
\sigma, \tau \in S_{n}}} \theta(\sigma) \theta(\tau) \\
& \leqq M \sum_{|\psi| \leqq n} \theta(\psi)^{2} \mathbf{P}[K(\psi)]^{-1} .
\end{aligned}
$$

In view of (3.3), $\mathbf{E}\left[X_{n}^{2}\right]$ is bounded, whence $\left\{X_{n}\right\}$ is uniformly integrable. Since $X_{n} \rightarrow 0$ a.s., it follows that $X_{n} \rightarrow 0$ in $L^{1}$ [DM, p. 23], which contradicts the fact that $\mathbf{E}\left[X_{n}\right]=1$.

\section{Spin Percolation}

Markov random fields lead naturally to quasi-Bernoulli percolations, as we shall see below. Thus, the critical temperature for spin percolation is amenable to calculation via Theorem 3.1. Because of its greater simplicity, we shall treat the case of constant interaction strength first.

Theorem 4.1. Let $\Gamma$ be a tree. The critical temperature for percolation in the Ising model on $\Gamma$ with constant interaction strength $J>0$ is

$$
T_{p c}= \begin{cases}\frac{J}{k \operatorname{coth}^{-1} \frac{\mathrm{br} \Gamma}{2-\mathrm{br} \Gamma}} & \text { if } \text { br } \Gamma<2, \\ +\infty & \text { if } \text { br } \Gamma \geqq 2 .\end{cases}
$$

If $\gamma=\alpha /(\alpha+1)$, then (4.1) is the same as

$$
\gamma_{p c}=\max \left((\operatorname{br} \Gamma)^{-1}, 1 / 2\right)
$$

Proof. To the Gibbs state $(\Omega, \mathbf{P})$ on $\Gamma$, we associate the random subgraphs $\Gamma(\omega)=\left\{\sigma \in \Gamma: u_{\sigma}(\omega)=+1\right\}$ (with $e(\sigma)$ present iff $u_{\sigma}(\omega)=u_{\sigma}(\omega)=+1$ ). By the Markov random field property of the Gibbs state and the fact that $\Gamma$ is a tree, we see that

$$
\mathbf{P}[K(\sigma) \cap K(\tau) \mid K(\sigma \wedge \tau)]=\mathbf{P}[K(\sigma) \mid K(\sigma \wedge \tau)] \cdot \mathbf{P}[K(\tau) \mid K(\sigma \wedge \tau)]
$$

Therefore $(\Gamma, \Omega, \mathbf{P})$ is a quasi-Bernoulli percolation process. Now

$$
\begin{aligned}
\mathbf{P}[K(\sigma)] & =\mathbf{P}\left[u_{0}=1\right] \prod_{0<\tau \leqq \sigma} \mathbf{P}[K(\tau) \mid K(\overleftarrow{\tau})]=\mathbf{P}\left[u_{0}=1\right] \prod_{0<\tau \leqq \sigma} \mathbf{P}\left[u_{\tau}=1 \mid K(\overleftarrow{\tau})\right] \\
& =\mathbf{P}\left[u_{0}=1\right] \prod_{0<\tau \leqq \sigma} \mathbf{P}\left[u_{\tau}=1 \mid u_{\tau}=1\right] ;
\end{aligned}
$$


in the last step, we have again used the Markov random field property. To calculate $\mathbf{P}\left[u_{\tau}=1 \mid u_{\tau}=1\right]$, it suffices to consider the tree $\Gamma^{\tau} \cup\{\overleftarrow{\tau}\}$ rooted at $\overleftarrow{\tau}$. Now it is easy to see that

Consequently

$$
\frac{\mathbf{P}\left[u_{\bar{\tau}}=u_{\tau}=1\right]}{\mathbf{P}\left[u_{\bar{\tau}}=1 \& u_{\tau}=-1\right]}=\alpha f(\tau)
$$

which gives us

$$
\mathbf{P}\left[u_{\tau}=1 \mid u_{\tau}=1\right]=\frac{\alpha f(\tau)}{\alpha f(\tau)+1},
$$

$$
\mathbf{P}[K(\sigma)]=\frac{f(0)}{f(0)+1} \prod_{0<\tau \leqq \sigma} \frac{\alpha f(\tau)}{\alpha f(\tau)+1} .
$$

For $T$ larger than the value for $T_{p c}$ asserted in (4.1), we have $T>T_{c}$, whence $f \equiv 1$ and the above reduces to $\frac{1}{2} \gamma^{|\sigma|}$. Thus, if $\gamma \cdot$ br $\Gamma<1$, Theorem 3.1 ensures that the probability of an infinite cluster of up spins containing 0 is 0 . Since $T>T_{c}$, the same is true of down spins, and since $\Gamma$ is connected, this entails the a.s. lack of any infinite cluster.

On the other hand, if $T$ is smaller than the value for $T_{p c}$ asserted in (4.1), then since $f \geqq 1$, we still have that $\mathbf{P}[K(\sigma)] \geqq \frac{1}{2} \gamma^{|\sigma|}$. Now Theorem 3.1 provides that 0 is in an infinite cluster with positive probability. Since the tail field is trivial for extreme Gibbs states [Pr2, Theorem 2.1], this guarantees the a.s. existence of some infinite cluster.

As before, this leads to the determination of the value of $T_{p c}$ for the diluted Ising model (by means of Corollary 6.3 of [Lyo]).

Corollary 4.2. Let $\Gamma$ be a tree, $(\text { br } \Gamma)^{-1}<p \leqq 1$, and $J>0$. For the diluted Ising model on $\Gamma$ with survival probability $p$ and constant interaction strength $J$, we have a.s.

$$
T_{p c}(p)= \begin{cases}\frac{J}{k \operatorname{coth}^{-1}\left(\frac{p \cdot \operatorname{br} \Gamma}{2-p \cdot \operatorname{br} \Gamma}\right)} & \text { if } p \cdot \operatorname{br} \Gamma<2, \\ +\infty & \text { if } p \cdot \operatorname{br} \Gamma \geqq 2 .\end{cases}
$$

We now generalize to varying interaction strengths. In fact, it is no more difficult to handle the case of random interaction strengths immediately.

Proof of Theorem 1.2. Let the probability space corresponding to the random variables $\left\{J_{\sigma}\right\}$ be $\left(\Omega^{\prime}, \mathbf{P}\right)$ and that corresponding to the Gibbs state associated with $\left\{J_{\sigma}\left(\omega^{\prime}\right)\right\}$ be $\left(\Omega, \mathbf{P}_{\omega^{\prime}}\right)\left(\omega^{\prime} \in \Omega^{\prime}\right)$. The compound process yields the random subgraphs $\Gamma\left(\omega^{\prime}, \omega\right)$, as in the preceding proof, representing the clusters of plus spins. For each $\omega^{\prime},\left(\Gamma\left(\omega^{\prime}, \cdot\right), \Omega, \mathbf{P}_{\omega^{\prime}}\right)$ is a quasi-Bernoulli percolation process with

$$
\mathbf{P}_{\omega^{\prime}}[K(\sigma)]=\frac{f_{\omega^{\prime}}(0)}{f_{\omega^{\prime}}(0)+1} \prod_{0<\tau \leqq \sigma}\left(1+\alpha_{\tau}\left(\omega^{\prime}\right)^{-1} f_{\omega^{\prime}}(\tau)^{-1}\right)^{-1} .
$$

If $\underset{\Pi}{\inf } \mathbf{E}\left[\sum_{\sigma \in \Pi} \prod_{0<\tau \leqq \sigma}\left(1+\alpha_{\tau}^{-1}\right)^{-1}\right]=0$, then by Fatou's lemma, 


$$
\inf _{\Pi} \sum_{\sigma \in \Pi} \prod_{0<\tau \leqq \sigma}\left(1+\alpha_{\tau}^{-1}\right)^{-1}=0 \quad \text { a.s. }
$$

Furthermore, by Theorem $1.1, T>T_{c}$ a.s., so that $f_{\omega^{\prime}} \equiv 1$ a.s. This means that (3.1) holds and percolation does not occur a.s.

On the other hand, if $T$ is less than the asserted value of $T_{p c}$, choose $T^{\prime}>T$ such that $\inf _{\Pi} \mathbf{E}\left[\sum_{\sigma \in \Pi} \prod_{0<\tau \leqq \sigma}\left(1+\alpha_{\tau}^{-1}\right)^{-1}\right]>0$ and set $\alpha_{\sigma}^{\prime}=e^{2 J_{\sigma} /\left(k T^{\prime}\right)}$. Now

$$
\left(1+\left(\alpha_{\sigma}^{\prime}\right)^{-1}\right)^{-1}\left(1+\alpha_{\sigma}^{-1} f(\sigma)^{-1}\right) \leqq \frac{1+\alpha_{\sigma}^{-1}}{1+\left(\alpha_{\sigma}^{\prime}\right)^{-1}} \leqq e^{-\varepsilon\left(1 / T-1 / T^{\prime}\right)},
$$

where

$$
\varepsilon=\inf \left\{\frac{d}{d t} \log \left(1+e^{-2 J \sigma^{t / k}}\right)^{-1}: \frac{1}{T^{\prime}} \leqq t \leqq \frac{1}{T}, 0 \neq \sigma \in \Gamma\right\}
$$

is positive a.s. in view of the hypotheses. Let

$$
w_{n}=e^{-n \varepsilon\left(1 / T-1 / T^{\prime}\right)} .
$$

Then $\sum_{n} w_{n}<\infty$ a.s. and

$$
\inf _{\Pi} \sum_{\sigma \in \Pi} w_{|\sigma|} \mathbf{P}[K(\sigma)] \geqq \inf _{\Pi} \sum_{\sigma \in \Pi} \frac{1}{2} \prod_{0<\tau \leqq \sigma}\left(1+e^{-2 J_{\tau} /\left(k T^{\prime}\right)}\right)^{-1}>0,
$$

whence Theorem 3.1 ensures percolation with positive probability a.s. In fact, percolation is a.s. as before.

\section{Bernoulli Percolation on Tree-like Graphs}

From every tree-like graph, $G$, and cutpoint $0 \in G$, we form a tree, $\Gamma$, rooted at 0 in the following manner. The vertices of $\Gamma$ are the cutpoints of $G$ and $\sigma$ has $\tau$ as a successor iff $\sigma$ and $\tau$ belong to the same block and every path in $G$ from 0 to $\tau$ passes through $\sigma$. Every subgraph $G^{\prime}$ of $G$ induces a subgraph $\Gamma^{\prime}$ of $\Gamma$ as follows: a vertex $\sigma \in \Gamma$ lies in $\Gamma^{\prime}$ iff $\sigma \in G^{\prime}$ and an edge in $\Gamma$ lies in $\Gamma^{\prime}$ iff its endpoints lie in $G^{\prime}$ and are connected in $G^{\prime}$. In particular, every bond or site percolation process on $G$ induces one on $\Gamma$; if that on $G$ is Bernoulli, then that on $\Gamma$ is quasi-Bernoulli in many cases-e.g., if the blocks of $G$ are uniformly bounded in size.

Theorem 5.1. Let $G$ be a tree-like graph, $0 \in G$ a cutpoint, and $\Gamma$ the associated tree. Suppose that for each $0 \leqq p<1$, there are constants $M<\infty$ and $r<1$ such that if $\sigma, \tau \in \Gamma \backslash\{0\}, \overleftarrow{\sigma}=\overleftarrow{\tau}, \sigma \neq \tau$, and $\sigma$ and $\tau$ are in the same block $B$ in $G$, then

$$
\mathbf{P}_{p}[\sigma, \tau, \overleftarrow{\sigma} \text { are connected }] \leqq M \mathbf{P}_{p}[\sigma, \overleftarrow{\sigma} \text { are connected }] \mathbf{P}_{p}[\tau, \overleftarrow{\sigma} \text { are connected }]
$$

and

$$
s_{\sigma}(p):=\mathbf{P}_{p}[\sigma, \overleftarrow{\sigma} \text { are connected }] \leqq r
$$


where $\mathbf{P}_{p}$ denotes probability with respect to Bernoulli bond [site] percolation on $B$ with survival parameter $p$. Then the critical probability for Bernoulli bond [site] percolation on $G$ is given by

$$
p_{c}(G)=\sup \left\{p: \inf \sum_{\Pi \in \Pi} \prod_{0<\tau \leqq \sigma} s_{\tau}(p)=0\right\},
$$

where $\Pi$ denotes a cutset of $\Gamma$.

It follows that $p_{c}(G)=p_{\text {cut }}(G)$ (for both bond and site percolation on $G$ ) because cutsets $\Pi$ of $\Gamma$ give only some of the cutsets of $G$. Note that the hypotheses are satisfied if the blocks of $G$ are uniformly bounded in size.

Proof. The condition (5.1) guarantees that Bernoulli percolation on $G$ induces quasi-Bernoulli percolation on $\Gamma$. Furthermore, in the notation of Sect. 3, $\mathbf{P}[K(\sigma)]=\prod_{0<\tau \leqq \sigma} s_{\tau}(p)$. Thus, in light of Theorem 3.1, it suffices to show that for $p<p^{\prime}<1$,

$$
\sum_{n \geqq 1} \sup _{|\sigma|=n} \prod_{0<\tau \leqq \sigma} s_{\tau}(p) / s_{\tau}\left(p^{\prime}\right)<\infty,
$$

which we shall accomplish by demonstrating that

$$
\sup _{0 \neq \sigma \in \Gamma} s_{\sigma}(p) / s_{\sigma}\left(p^{\prime}\right)<1 .
$$

Now the law of $\boldsymbol{B}(\omega)$ under $\mathbf{P}_{\boldsymbol{p}_{1} p_{2}}$ is the same as the law of $B\left(\omega_{1}\right) \cap B\left(\omega_{2}\right)$, where $B\left(\omega_{i}\right)$ are chosen independently via $\mathbf{P}_{p_{i}}(i=1,2)$, respectively. Of course, if $\sigma$ and $\bar{\sigma}$ are connected in $B\left(\omega_{1}\right) \cap B\left(\omega_{2}\right)$, then they are connected in each of $B\left(\omega_{i}\right)$. Therefore

$$
s_{\sigma}\left(p_{1} p_{2}\right) \leqq s_{\sigma}\left(p_{1}\right) \cdot s_{\sigma}\left(p_{2}\right) .
$$

It follows that $s_{\sigma}(p) / s_{\sigma}\left(p^{\prime}\right) \leqq s_{\sigma}\left(p / p^{\prime}\right)$, which, together with (5.2), completes the proof.

\section{References}

[AB] Aizenman, M., Barsky, D. J.: Sharpness of the phase transition in percolation models. Commun. Math. Phys. 108, 489-526 (1987)

[Big] Biggs, N. L.: Interaction Models. Cambridge: Cambridge University Press 1977

[Bis1] Bishop, A. R.: Percolation theory and the Ising model. J. Phys. C: Solid St. Phys. 6, 2089-2093 (1973)

[Bis2] : Percolation theory and the Ising model for a class of triangular cactii. Prog. Theor. Phys. 52, 1798-1806 (1974)

[Bis3] _ _ A use of Bethe lattice solutions for percolation-constrained phenomena. Prog. Theor. Phys. 53, 50-65 (1975)

[Con] Coniglio, A.: Some cluster-size and percolation problems for interacting spins. Phys. Rev. B 13, 2194-2207 (1976)

[DM] Dellacherie, C., Meyer, P.-A.: Probabilities and Potential. Amsterdam: North Holland 1978

[FE] Fisher, M. E., Essam, J. W.: Some cluster size and percolation problems. J. Math. Phys. 2, 609-619 (1961) 
[Har] Harary, F.: Graph Theory. Reading, MA: Addison-Wesley 1969

[KM] Katsura, S., Matsubara, F.: Magnetic properties of the random mixture of Ising spins. Can. J. Phy. 52, 120-130 (1974)

[KT] Katsura, S., Takizawa, M.: Bethe lattice and Bethe approximation. Prog. Theor. Phys. 51, 82-98 (1974)

[KS] Kindermann, R., Snell, J. L.: Markov Random Fields and their Applications. Providence, RI: American Mathematical Society 1980

[Lig] Liggett, T. M.: Interacting Particle Systems. Berlin, Heidelberg, New York: Springer 1985

[Lyo] Lyons, R.: Random walks and percolation on trees. Ann. Probab. (to appear)

[Mtb] Matsubara, F.: Magnetic properties of random mixture of the one-dimensional Ising chain. Prog. Theor. Phys. 51, 378-390 (1974)

[Mtd] Matsuda, H.: Infinite susceptibility without spontaneous magnetization-exact properties of the Ising model on the Cayley tree. Prog. Theor. Phys. 51, 1053-1063 (1974)

[MMS] Menshikov, M. V., Molchanov, S. A., Sidorenko, A. F.: Percolation theory and some applications. (In Russian), Itogi Nauki i Tekhniki (Series of Probability Theory, Mathematical Statistics, Theoretical Cybernetics) 24, 53-110 (1986); J. Soviet Math. 42, 1766-1810 (1988); (English translation)

[MS] Moore, T., Snell, J. L.: A branching process showing phase transition. J. Appl. Prob. 16, 252-260 (1979)

[Pr1] Preston, C. J.: Gibbs States on Countable Sets. Cambridge: Cambridge University Press 1974

[Pr2] ___ Random Fields. Lecture Notes in Math., vol. 534. Berlin, Heidelberg, New York: Springer 1976

[Spi] Spitzer, F.: Markov random fields on an infinite tree. Ann. Probab. 3, 387-398 (1975)

Communicated by M. E. Fisher

Received October 7, 1988; in revised form March 28, 1989 
